\title{
Informational interaction service-simulating test implementation peculiarities of the Internet of things
}

\author{
O.V. Selishchev ${ }^{1, *}$, T.A. Zolotareva ${ }^{1}$, and I.V. Voronin ${ }^{1}$ \\ ${ }^{1}$ Lipetsk State Pedagogical P.Semenov-Tyan-Shansky University, Lipetsk, 398020, Russia
}

\begin{abstract}
This article discusses the technology of building sensor networks. On the basis of the article there is an example of the servicesimulating test for the informational interaction of the Internet of things. The probabilistic-and-time characteristic of the informational interaction of the Internet of things is evaluated. This factor enhances the quality of computer network service. The article also considers a number of tasks, allowing us to build optimal modes of sensor networks functioning. A probabilistic model that allows us to evaluate absolute and probabilistic characteristics of informational interaction is built.
\end{abstract}

\section{Introduction}

Nowadays the Internet of things technology is gaining more and more popularity. This technology originates from dispersed sensor networks, which were initially developing as local sensor networks, and later they moved to the global Internet networks. Sensor networks are dispersed networks consisting of small wireless nodes of a narrow specialization (sensor devices). A great number of these networks is spread in a certain area. It means that they are based on the collaboration of a large number of miniature nodes [1].

The advantage of service-simulating test is that you can replace the changing of events process with the accelerated changing of events process in the system during the program operation in real time. As a result, you can process the operation of the simulated system in a few minutes and evaluate this operation in a wide range of variable parameters [2].

During the service-simulating test, theoretical and real critical situations, real processes are also operated, and special cases are studied. The main advantage of the service-simulating test is the ability to carry out various experiments with the object under study without employing physical implementation. It allows us to predict and prevent a large number of unexpected situations which can lead to unjustified costs during the operation [3].

\footnotetext{
*Corresponding author: ole9153@yandex.ru
} 


\section{The technology of building the internet of things networks}

The sensor device consists of four main components (see figure 1). They include power supply, data transmission, collecting and processing data.
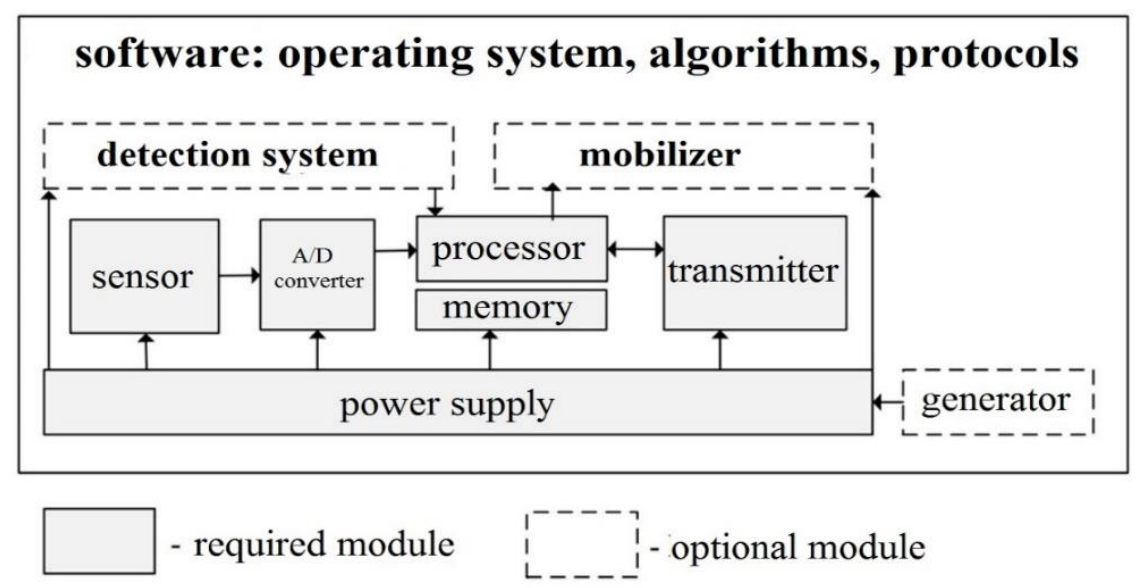

Fig. 1. Components of sensor devices.

A number of compulsory requirements are applied to sensor devices. They should:

- have low energy consumption, as well as low cost price;

- interact with a large number of the same sensor devices at short distances;

- work in an offline mode;

- adapt to the environment.

\section{Evaluation of the probabilistic-and-time characteristics of the informational interaction}

Depending on the carrying out tasks, the number of sensor devices in networks can range from several dozens to several thousands. High density and limited bandwidth networks should provide high-quality services.

Unlike the ordinary network, the sensor network consists of a large number of sensor nodes. These nodes may fail during the operation, have insufficient power supply, or be exposed to external critical conditions. Failure of one or more sensor nodes should not affect the overall performance of the network or its parts.

The computer network quality of service is expressed in probabilistic-and-time characteristics. For the Internet of things network this characteristic is expressed as the following sum:

$$
t_{i}=t_{c}+t_{d}
$$

where ti. - is the time of the informational interaction;

tc. - is the time required to the connection of the informational interaction;

td. - is the time of data transmission via the setting connection of the informational interaction. 


\section{The implementation of the service-simulating test for connection of installation}

Time of the informational impact may vary due to some factors: there are may be multiple changes in the network topology due to the appearance of new nodes or disconnection of existing ones, and besides there are some critical changes of the external environment. These factors should be considered while developing a connection model for the informational interaction [4].

Thus, the installation of the informational interaction is the building of a logical channel. This channel connects the destination gateway with the sensor device for subsequent data transmission. Due to the mesh-topology of the Internet of things network there may be several logical channels which connect the destination gateway and the device (see figure 2).

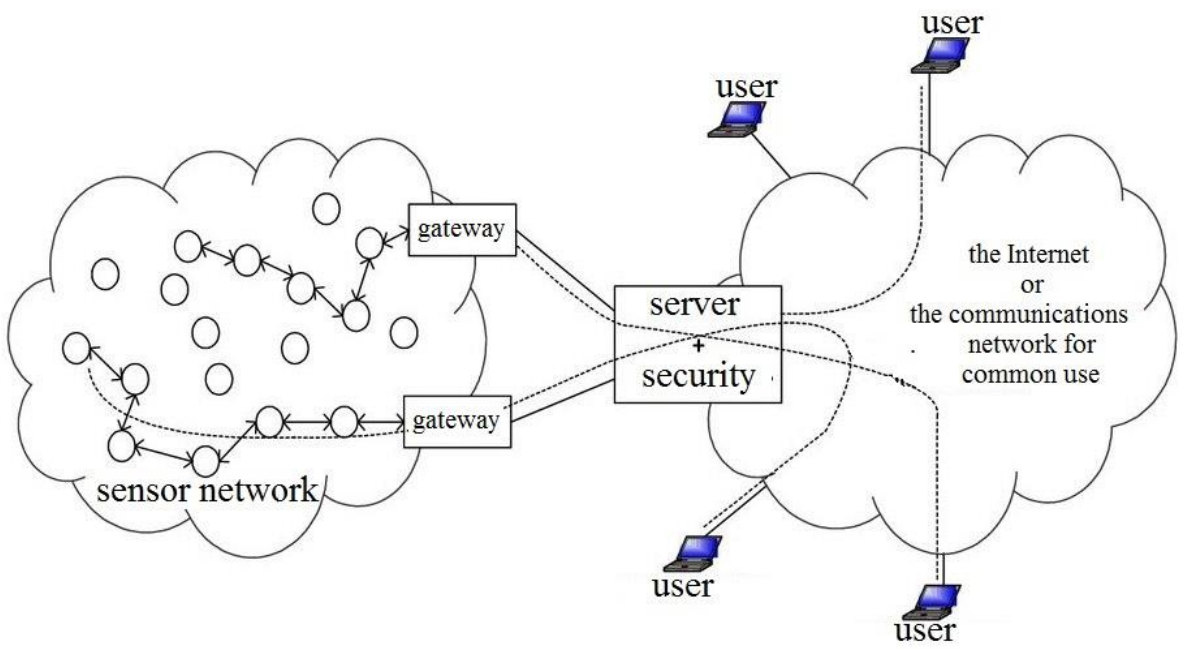

Fig. 2. Logical channels for data transmission from the sensor device to the cloud.

Some repeated attempts of connection of the sensor device to the network may occur. Due to this fact, we define time of the installation of the informational interaction (tf) as a random value that can be found by the following formula:

$$
t_{f}=\sum_{i=1}^{n_{0}} t_{o_{i}}+\sum_{i=1}^{n_{k}} t_{k_{i}}+n_{a} t_{n}
$$

where no - is the number of physical channels which the signal returned to while searching for an alternative logical channel;

$\mathrm{nk}$ - is the number of physical channels in the logical one (logical channel is built from the source sensor device to the destination sensor device);

na - is the number of attempts to install the informational interaction;

$t_{o_{i}}$-reverse time passing of the physical i-channel;

$t_{k_{i}}$ - time passing of the physical i-channel;

tII - time of switching to the other logical channel.

The calculated quantity of tf. shows the result of the installed informational interaction:

- if tf. $\leq$ tpermissible, then the informational interaction is installed;

- if tf. $>$ tpermissible, then the informational interaction is installed with a low quality of service, which is critical for the urgent delivery data [5];

- if $\mathrm{n} \Pi>$ npermissible, then the connection is not installed. 
Thus, the initial data for the informational interaction modeling are:

- transmission time of the forward and reverse signal via the physical channel;

- allowable time and number of attempts to install the informational interaction;

- time of reconnection to the logical channel.

Among the great number of logical channels Lij, there is their ordering according to the number of occupied physical c-channels $(\mathrm{c}=\mathrm{cmin}, \ldots$, cmax). In the set of Lij, a number of $\mathrm{c}-$ values (for occupied physical channels) is raffled off. As a result there is a process of a signal passing from the source $\mathrm{i}$ to the destination $\mathrm{j}$. The procedure is repeated $\mathrm{N}$-quantity times. According to the results of experiments, we can calculate average time for the informational interaction installation.

The number of lost physical c-channels leads to a random channel number out of vpossible $(\mathrm{c} \in \mathrm{v})$. The next number of the lost physical w-channel is found by the formula $\mathrm{w}=/ \mathrm{Uv}+1 /$, where $\mathrm{U}$ is a random number and $\mathrm{U} \in[0,1]$ (it is calculated by the random-number generator). Brackets / / mean the rounding numbers down. "1"-value is given to the physical channel with the w-number in the set of logical channels (Lij). The procedure for wdetermining is repeated c-quantity times.

The calculation of the characteristics of the informational interaction installation can be led to the evaluation of the mathematical expectation $\mathrm{M} \xi$-discrete random value $\xi=\mathrm{f}(\alpha)$, where $\alpha=(\alpha 1, \ldots, \alpha)$ has the probability distribution $\mathrm{p}(\alpha \sim \mathrm{p})$, which is known. The random $\xi$-value has two meanings $\xi \in\{0,1\}$, where:

$\xi=1-$ connection is installed;

$\xi=1-$ connection is not installed.

The value $\mathrm{M} \xi=\mathrm{P}\{\xi=1\}$ means the probability of the connection failure. The random $\xi$ value is not clear, in other words it means $0<\mathrm{M} \xi<1$.

Imagine a random $\alpha$-value as a vector $\alpha=\left(\alpha_{1}^{v}, \ldots, \alpha_{d}^{v}\right)$. It shows the state of the physical channels which are included in the simulated set of logical channels Lij according to their status: whether they exist or not. The random value $\alpha_{i}^{v} \in\{0,1\}, i=\overline{1, d}$ (where $\mathrm{d}$ is the number of physical channels in the set of Lij). The value $\alpha_{i}^{v}=1$ means the loss of the physical $\mathrm{i}$-channel at the speed of v-value. Random values $\alpha_{i}^{v}$ are independent, $\alpha$ has a finite set of values $\alpha \in X, X=\left\{x_{j} ; j=1, \ldots, n ; n=2^{d}\right\}$. For these values there is a distribution $\rho(\mathrm{x}), x \in X$ is given by the set of the following probabilities $\rho\left(x_{j}\right)=P\left\{\alpha=x_{j}\right\}=p_{j} \geq$ $0, j=\overline{1, n}$. Considering the classification of the set of logical channels (Lij) by the number of occupied physical channels (c), the mathematical expectation evaluation can be found by the following formula:

$$
\widetilde{M} \xi=\sum_{c_{\min }}^{c_{\max } \Sigma} M \xi(c)
$$

where $\widetilde{M} \xi$ is the probability evaluation of the informational interaction failure with the existence of physical c-channels that do not carry out a signal.

You can establish Lij(c)-states during the k-draw by applying a random selection of physical channels-c numbers. These channels do not carry out signals to determine the informational interaction. As a result, the $\alpha$-vector gets a concrete x-implementation, containing c-units and (d-c)-zeros. According to the rule of the informational interaction installation and the rule of carrying out a signal through the set of alternative logical channels Lij the value is calculated by the following formula:

$$
\xi(x \mid c)_{k}=f\left[L_{i j}^{k}\right]
$$

where $\xi(x \mid c)_{k}$-is the result of the installation (or the failure) of the informational interaction with the k-test (k-implementation of Lij), $\xi(x \mid c)_{k} \in(0,1)$, and the probability of the obtained implementation is 


$$
p(x)=p\left[L_{i j}^{k}\right]
$$

This value $\xi(x \mid c)_{k}=1$ occurs if the signal does not reach the destination (which means that none of logical channels are built out of the set of Lij). Otherwise it can occur if the number of attempts exceeds the allowable value, or the signal reaches the destination and the informational interaction is installed, but without proper quality of service.

The following formula helps to evaluate the probability of the informational interaction failure between sensor devices (in the case of losing physical c-channels in the set of all logical channels (Lij):

$$
\widetilde{M} \xi(c)=\frac{c_{d}^{c}}{N_{2 c}} \sum_{k=1}^{N_{2 c}} p\left(L_{i j}^{k} \mid \xi(x \mid c)=1\right)
$$

The probability $\mathrm{p}(\mathrm{Lij})$ is found by the following formula:

$$
p\left(L_{i j}^{k}\right)=\prod_{i=1}^{h} p\left(\alpha_{i}=1\right) \prod_{j=1}^{d-h}\left[1-p\left(\alpha_{i}=1\right)\right]
$$

This formula completely reflects the probabilistic approach to the informational interaction installation in the Internet of things.

\section{Conclusion}

This article shows that the Internet of things technology building is closely related to a number of tasks which require modeling of the informational interaction processes. It allows us to build optimal operation modes of such systems.

The probabilistic model of the informational interaction installation in the Internet of things is considered. This model provides conditions that correspond to the actual process of informational interaction. This factor includes such conditions as a limited number of reconnection attempts, the existence of non-functional access points and channels, and the existence of alternative routes. The model allows us to evaluate the absolute and probabilistic characteristics of the informational interaction. This model can be used in the Internet of things networks design at the early stages.

\section{References}

1. Akyildiz I F 2006 Key Wireless Networking Technologies in the Next Decade. NEW2AN 2006 (St. Petersburg: Keynote Speech)

2. Ryzhikov Yu I 2015 Imitatsionnoe modelirovanie. Teoriya i tekhnologiya ( $\mathrm{SPb}$ : KORONA print)

3. Sovetov B Ya, Yakovlev S A 2015 Modelirovanie sistem (M.: Yurait) p 343

4. Vinel A A 2008 GLOBECOM Workshops 2008 IEEE 41

5. Gol'dshtein B S, Kucheryavyi A E 2014 Seti svyazi post-NGN (SPb.: BKHV-Peterburg) 